\title{
Analysis and simulation of the IEC 61850 standard reliability
}

\author{
Oscar D. Flórez Cediel ${ }^{\# 1}$, Julián R. Camargo López ${ }^{\# 2}$, Danilo A. López Sarmiento ${ }^{\# *}$ \\ ${ }^{\# 1}$ Full Time Professor at Universidad Distrital Francisco José de Caldas, \\ Faculty of Engineering, Bogotá (Colombia-South America) \\ odflorez@udistrital.edu.co

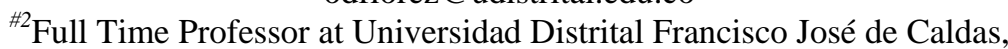 \\ Faculty of Engineering, Bogotá (Colombia-South America) \\ jcamargo@udistrital.edu.co \\ \#3 Full Time Professor at Universidad Distrital Francisco José de Caldas, \\ Faculty of Engineering, Bogotá (Colombia-South America) \\ dalopezs@udistrital.edu.co
}

\begin{abstract}
One of the important elements within an electrical system is the electrical substation since it is the sub-system that produces, transforms, regulates and distributes power. Ensuring that this process works properly is key in everything related to the energy industry; this document is an analysis and meticulous study that focuses on comparing the calculation of the reliability of a typical electrical substation, with and without implementing the IEC61850 (GOOSE) standard. The results suggest that when implementing the IEC61850 standard, system reliability is increased by $15 \%$.
\end{abstract}

Keyword- Reliability, double busbar substation, IEC 61850, Monte Carlo simulation.

\section{INTRODUCTION}

Due to different faults that arise in a substation, it is necessary to estimate the system reliability. The method to calculate this value is the fault tree, to determine the losses that can be had in the substation.

According to studies that have been carried out, the advantages and/or disadvantages of standardization must be known for each of the elements that make up the system for this study.

\section{THEORETICAL FRAMEWORK}

A substation is a key element of an electric power system, to feed the industrial, commercial and residential sectors, thus, it is necessary to incorporate a communications system to improve reliability in substation operations. Thus reducing downtimes to increase service quality, and diminishing economic losses.To calculate reliability, it is necessary to incorporate the fault tree to determine it and to predict possible problems in the study system; and in this manner, study the implementation of the IEC61850 standard (GOOSE).GOOSE (Generic Object Oriented Substation Event), it is a standard for fast transmission of events in a substation, such as commands, alarms, indications, as well as messages [1].Using LAN (Local Area Network) high-speed data transmission [2], decreases considerably the amount of wiring, and enables, because of its immunity to electromagnetic interference (in the case of fiber optics), its used as close as possible to the primary process.On the other hand, the use of IEDs, (Intelligent Electronic Device) based on microprocessors, offers new possibilities such as self-supervision, signal analysis, computational facilities for protection and control algorithms, data storage, event handling and fault analysis.Technological developments in this area have achieved a significant reduction in the physical space required to install protection, measurement, control and supervision systems, directly influencing a cost reduction in the project. Thereby improving the operation, reduction and maintenance planning, providing a number of benefits that are important advantages when compared to conventional systems.

\section{DEFINITIONS TO CALCULATE RELIABILITY}

Some definitions for calculating reliability are:

\section{A. Reliability}

It is defined as the probability that the system will perform its intended functions without incident for a certain period of time and under set conditions. Knowledge of reliability in electrical equipment is an important consideration in the design of power distribution systems for industrial plants. It is possible to make quantitative comparisons of the reliability between different designs, and use this information, with cost-benefit studies to determine which type of alternative should be used. [3]. 


\section{B. Fault Tree}

The Fault Tree (FTA) is a representation of the combination of events that cause the occurrence of an undesirable event. Este diagrama se realiza basándose en combinaciones definidas por operadores de tipo lógico, los cuales se presentan en la Fig. 1. El proceso deductivo continúa hasta que se llega a eventos básicos, los cuales son mutuamente independientes y tienen asociada una probabilidad de ocurrencia [4].

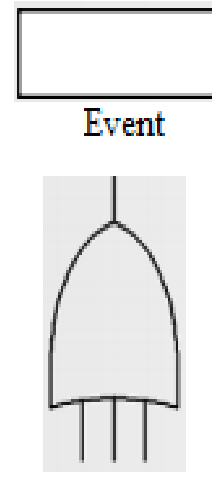

OR gate

\section{Event that can occur normally.}
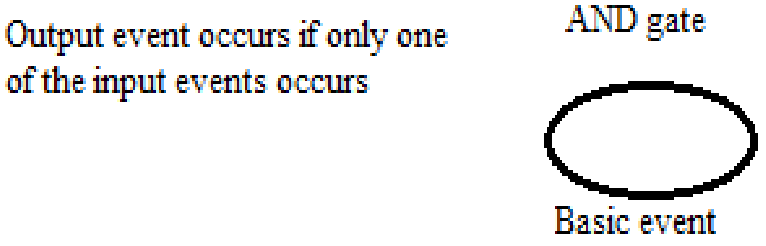

Outout event occurs if all input events occur simultaneously.

Basic event or failure.

Figure 1. Logical symbols of a FTA.

As a quantitative tool, the FTA is widely used because it reduces the possibility of the top event by determining the faults and errors that could cause the top event. Also, it allows analyzing the effects of the change or addition of components to a system, for example, the installation of a high-level alarm system.

\section{Substation}

The substation is comprised of a set of computers that control the flow of energy and ensure the safety of the system by automatic protective devices [5]. The configuration of a substation, is the arrangement of electromechanical equipment constituting a connection yard as long as these are referenced to the same voltage level. The reliability of a substation, depends on its protection system operation. The function of a protection system is to reduce the influence of a fault in the electrical system, so as not to affect the operation or the components of the substation, nor to endanger the life of human beings.

Taking a basic configuration of a substation, the unwanted event is the fault of the substation. Four basic events can be considered: failure in the transmission line module of the failure in the transformer module, failure in the Busbar module and failure in the circuit module. The occurrence of any of these basic fault events is represented by a type OR gate, the inputs being, each of the aforementioned events. The representation of the fault tree is presented in Fig. 2.

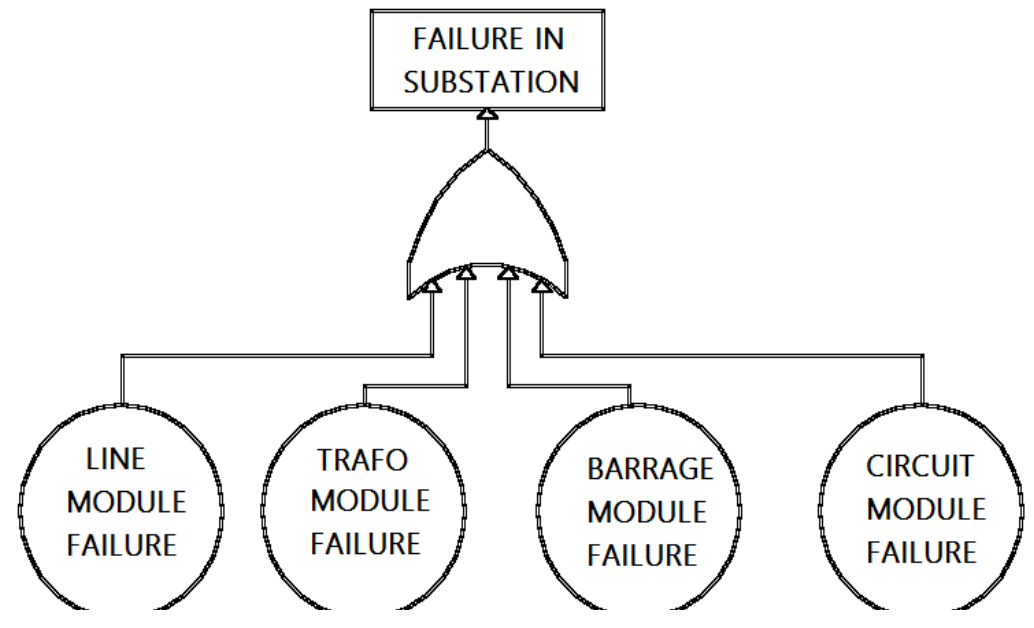

Figure 2. Fault tree of a substation. 


\section{RELIABILITY CALCULATION AND SIMULATION.}

To perform the reliability calculations, it should be noted that the Monte Carlo methods encompass a collection of techniques to obtain solutions of mathematical or physical problems through repeated random tests. In practice, random tests are replaced by results of certain calculations performed with random numbers [6]. Generally in statistics, random models are used to simulate phenomena that have some random component.

\section{A. Algorythm}

The Monte Carlo simulation algorithm developed is based on the generation of random numbers by the inverse transformation method, which is based on the cumulative frequency distributions for:

- Determine the random variables and their cumulative distributions (F).

- Generate a uniform random number between 0 y 1.

- Determine the value of random variables for the number generated according to classes that are had.

- Calculate mean, standard deviation error and perform the histogram.

- Analyze results for different sample sizes.

- Iterate as many times as samples are needed.

\section{B. Graphic interface}

To calculate reliability, a graphic interface was designed with the different modules that comprise the substation. To perform the corresponding program to calculate the reliability, using MATLAB GUI, the following general steps were taken into account:

- Data collection:

- Generation of random numbers.

- Values of failure rates and recovery times.

- Number of simulation interactions.

- Calculation of the first fault for each of the modules.

- Calculation of system failures.

- Calculation of recovery times of the system, until completing the determined number of interactions.

- Determination of the fault counter.

- Calculation of total system failures and determination of the percentage of system failure.

- Calculation of the operation and total operating time in system years.

- Calculation of recovery time and total recovery time in system hours.

- Calculation of total simulation time in hours.

- Determination of the percentage of failure time and system operation.

- Data collection:

* Power factor.

* Load factor.

* Value of KWh.

- Calculation of energy supplied (MWh-year).

- Cost of energy supplied (\$).

- Calculation of energy not supplied (MWh-year).

- Cost of energy not supplied (\$).

C. Analysis of the IEC61850 standard

The substation is formed by a set of computers that control the flow of energy and ensure system security with automatic protection devices [5].

The configuration of a substation is the arrangement of the electromechanical equipment constituting a connection yard and are referenced to the same voltage level; the representation diagram of a double bus substation is presented in Fig. 3. 


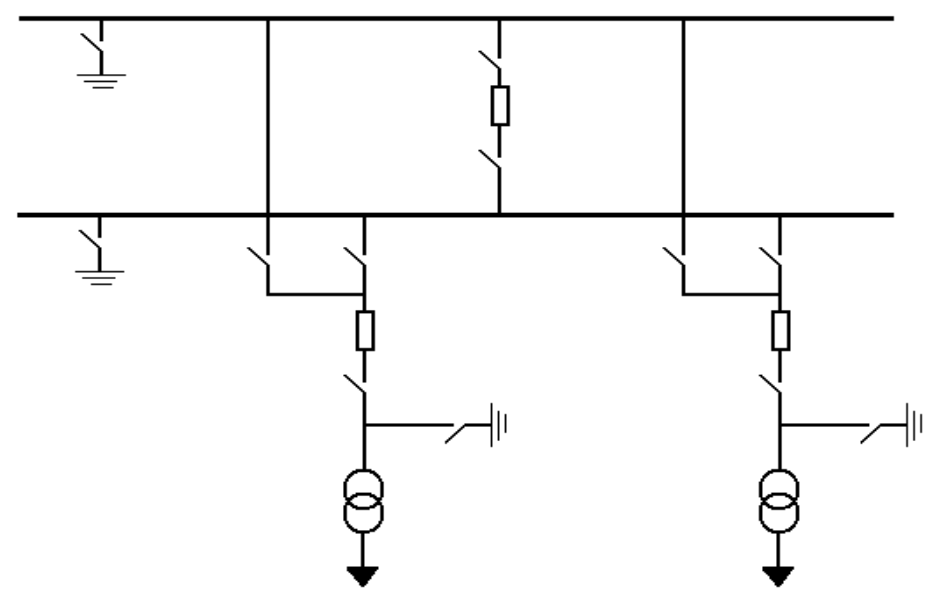

Figure 3. Configuration of a double bus substation.

The reliability of a substation depends on the operation of the substation's protection system. The function of a protection system is to reduce the influence of a failure in the electrical system, so that proper operation or components of the substation is not affected, nor jeopardize the lives of human beings [7]. Taking a basic configuration of a double bus substation, the unwanted event is the substation failure. They can be considered as basic events: failure in the transmission line module, failure in the transformer module, failure in the bus module, failure in the circuit module and the communications module. He occurrence of any of these basic fault events is represented by a type OR gate, the inputs being the same, each of the aforementioned events. The representation of the fault tree is presented in Fig. 4.

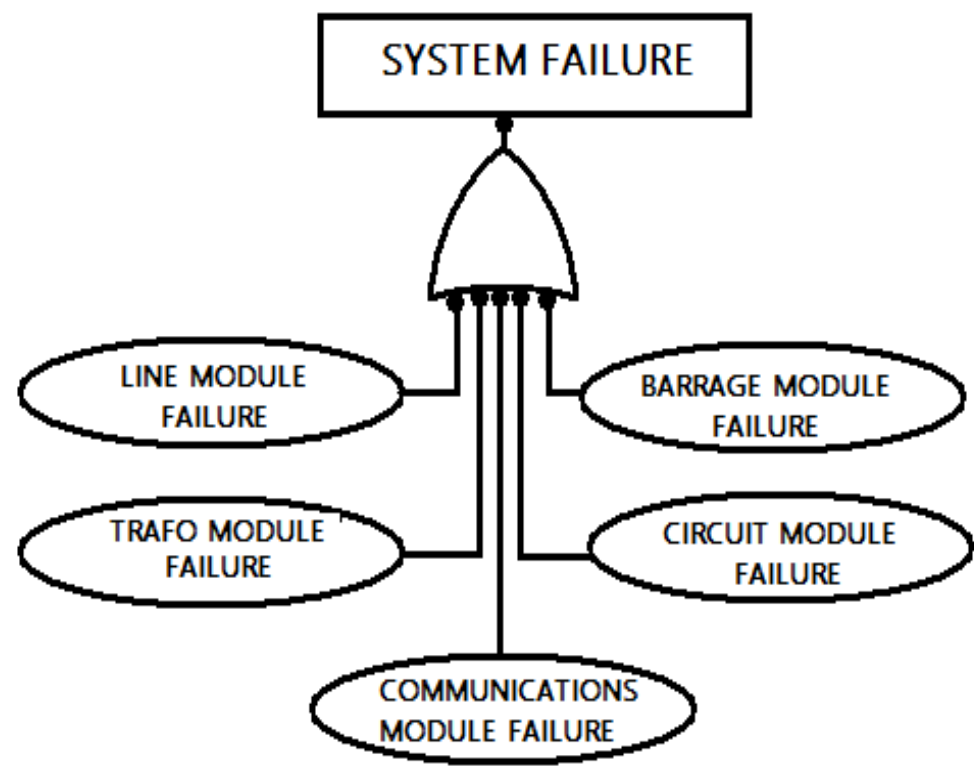

Figure 4. Configuration of a double barrage substation.

The use of high-speed LAN networks for data transmission considerably reduces the volume of cabling and, thanks to its immunity to electromagnetic interference (in the case of fiber optics), allows its use as close as possible to the primary process. On the other hand, the use of IEDs based on microprocessors, offers new possibilities such as self-supervision, signal analysis, computational facilities for protection and control algorithms, data storage, event handling and failure analysis.

The general operation of this standard is presented in accordance with the message that is transmitted from the control center to each of the substations, that is, from the control center the message is sent to each of the control stations, to be transmitted according to the coding established for each of the OMS, by means of electric cable and the TCP / IP protocol. 


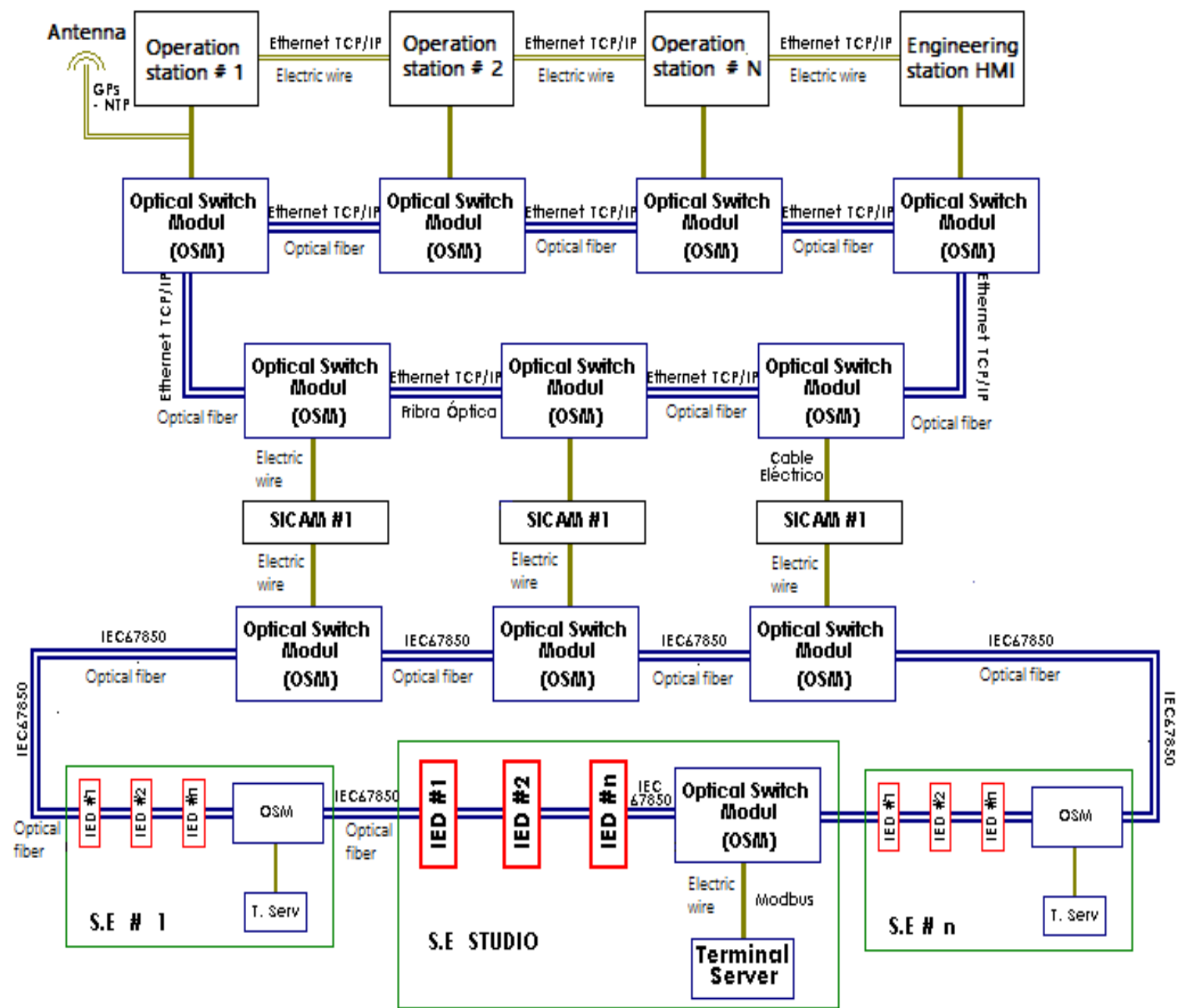

Fig. 5. Diagrama de control general de una subestación doble barraje con el estándar IEC61850.

Each one of the OMS that intervene in the process, communicate with each other by the TCP / IP protocol through Fiber Optics, to then communicate with the SICAM PASS which are in charge of converting the TCP / IP protocol to the IEC61850 protocol. The OSM and the IEDs of each of the substation, as shown in Fig. 5.

When this message is received by each of the OSM, it begins to transmit among the different OSM belonging to the substation by means of optical fiber, to be sent to all the IEDs that are communicated by this protocol. This being one of the advantages of the IEC61850 standard, since the message is transmitted to all the connected elements and the element that needs it, accepts them and decodes them for its use, delivering a received signal that is sent to the control center, and the element that is not affected by this message, ignores it.

The main disadvantage of this Protocol is in the operation by message transmission ring since if any part fails, it interrupts the transmission of the message that is sent from the substation main control center.

D. Simulations for calculating reliability

To perform the corresponding simulations of the substation operation under study, a graphic interface was implemented in MATLAB, as shown in Fig. 6. 


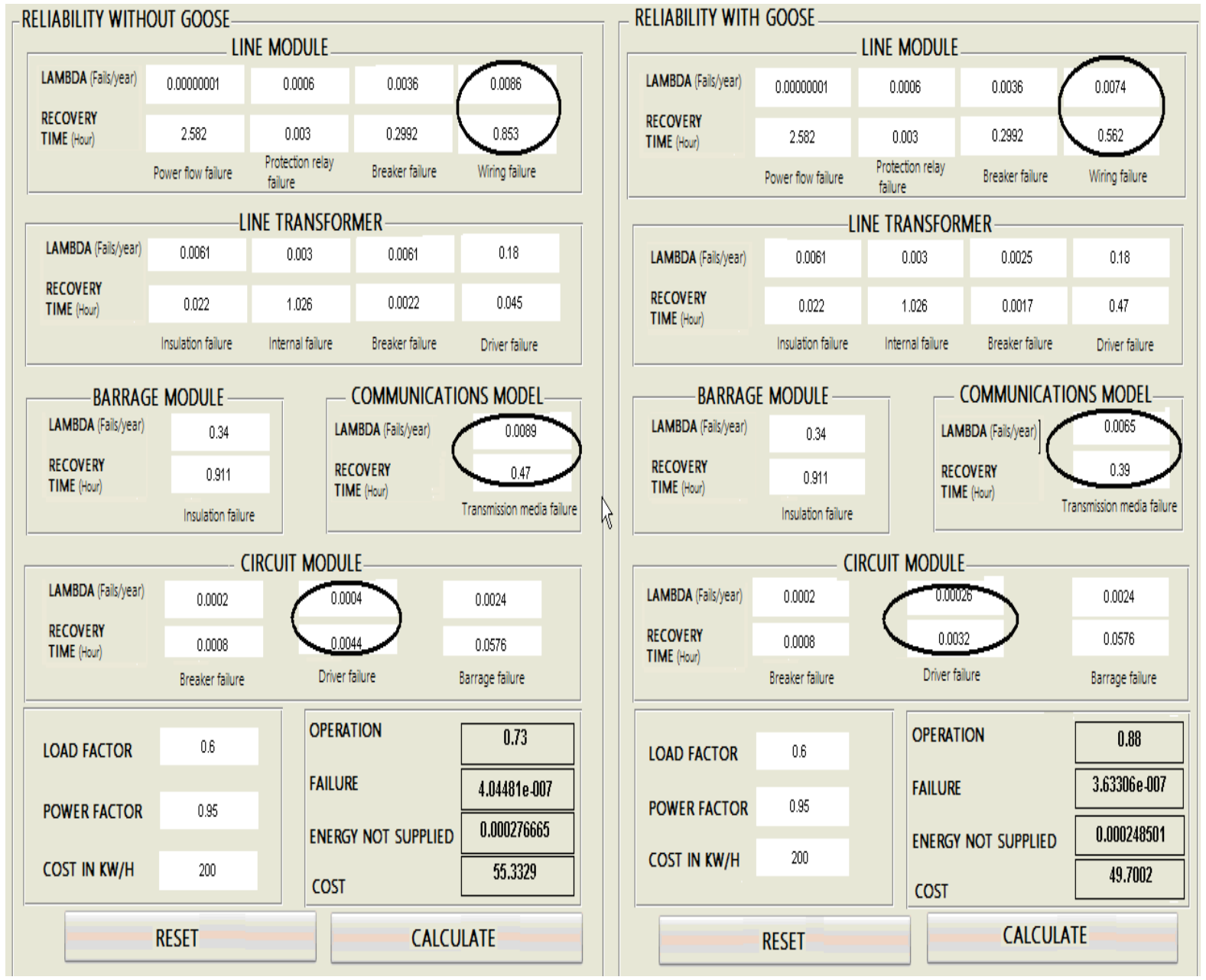

Figure 6. Graphic interface.

Each of the modules that are part of a typical double busbar substation is located in the interface, such as the line module, transformer module, bus module, communications module, circuit module, as well as the spaces for the load and power factors, in addition to the KWh cost.

In each of the modules, you should have the Lambda information and the recovery time where each of the system failures is presented, this information is obtained from international standards such as the GOLD BOOK and / or studies carried out to determine the reliability factors of the substation, as shown in Fig. 7 for the communications module applying the IEC61850 standard [8].

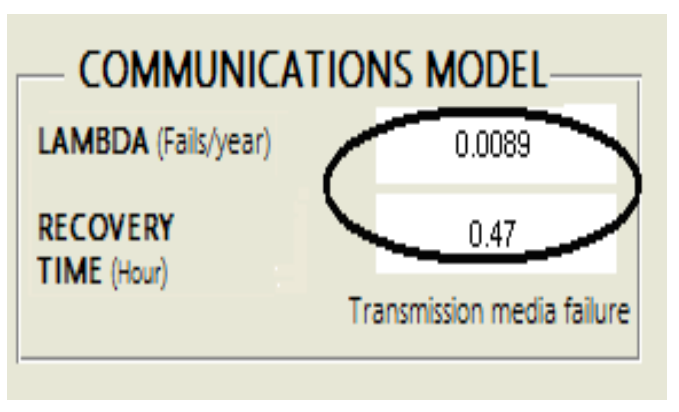

Figure 7. Communication module of the graphic interface.

According to studies conducted in this investigation, to implement the standard, the communications module is added, because this standard indicates the protocols that are used in substations when its implementation is done. Therefore, for the values in which differences are presented, we have in Table I, II and III, where the respective values are assigned to lambda and recovery time for the comparison without or with the standard. 
TABLE I. Comparison of interface results for the line module.

\begin{tabular}{|c|c|}
\hline \multicolumn{2}{|c|}{ Line Module } \\
\hline \multicolumn{2}{|c|}{ Cable failure } \\
\hline Without Goose & With Goose \\
\hline $\begin{array}{l}\text { Lambda }=0.0086 \text { Fail } / \text { Year } \\
\mathrm{T} \text { recovery }=0.853 \text { Hour }\end{array}$ & $\begin{array}{l}\text { Lambda }=0.0074 \text { Fail } / \text { Year } \\
\text { T recovery }=0.562 \text { Hour }\end{array}$ \\
\hline
\end{tabular}

TABLE II. Comparison of interface results for the communications module.

\begin{tabular}{|c|c|}
\hline \multicolumn{2}{|c|}{ Communications module } \\
\hline \multicolumn{2}{|c|}{ Communications failure } \\
\hline Without Goose & With Goose \\
\hline Lambda $=0.0089$ Fail/Year & Lambda $=0.0065$ Fail/Year \\
T recovery $=0.47$ Hour & recovery $=0.39$ Hour \\
\hline
\end{tabular}

TABLE III. Comparison of interface results for the circuit module.

\begin{tabular}{|c|c|}
\hline \multicolumn{2}{|c|}{ Circuit Module } \\
\hline \multicolumn{2}{|c|}{ Driver failure } \\
\hline Without Goose & With Goose \\
\hline $\begin{array}{c}\text { Lambda }=0.0004 \text { Fail/Year } \\
\text { T recovery }=0.0044 \text { Hour }\end{array}$ & $\begin{array}{c}\text { Lambda }=0.0026 \text { Fail/Year } \\
\text { T recovery }=0.0032 \text { Hour }\end{array}$ \\
\hline
\end{tabular}

D. Results of general system simulations

The results obtained from the simulations performed are those shown in Tables IV and V.

TABLE IV. Results of the simulations without GOOSE

\begin{tabular}{|l|c|}
\hline \multicolumn{2}{|c|}{ Results Without GOOSE } \\
\hline \% Functioning & $72 \%$ \\
\hline \% Fail & $28 \%$ \\
\hline Energy Supplied & 4.374 .000 \\
\hline Energy Not Supplied & 0,00049439 \\
\hline Cost E S & $\$ 874.810 .000 .000$ \\
\hline Cost E N S & $\$ 42,93$ \\
\hline
\end{tabular}

TABLE V. Results of the simulations with GOOSE

\begin{tabular}{|l|c|}
\hline \multicolumn{2}{|c|}{ Results With GOOSE } \\
\hline \% Functioning & $88 \%$ \\
\hline \% Fail & $12 \%$ \\
\hline Energy Supplied & 5.272 .800 \\
\hline Energy not Supplied & 0,00021467 \\
\hline Cost E S & $\$ 1.054 .600 .000 .000$ \\
\hline Cost E N S & $\$ 98,88$ \\
\hline
\end{tabular}




\section{E. Economic analysis}

To calculate the IRR, we look for the interest rate that makes the flow of money brought to present value equals zero (0). The interest rate at which this occurs is a measure of the totality of the benefits that the investment generates while it is in the project. The decision rules applied are:

- $\quad$ If the IRR is greater than the minimum acceptable rate, the project must be accepted.

- If the IRR is equal or similar to the minimum acceptable rate, the decision making is indifferent.

- $\quad$ If the IRR is less than the minimum acceptable rate (Opportunity Rate), the project must be rejected.

- $\quad$ The opportunity rate is understood as the current bank rate.

In the case of the analysis and simulation of the implementation of Standard IEC61850, the financial viability of the project is shown, since it shows that the IRR rate is greater than $1.98 \%$ E.A. with which the company would benefit, since it would increase the profitability of their services as well as their users with improved quality of service (see Table VI and VII).

TABLE VI. Economic analysis without GOOSE

\begin{tabular}{||l|c||}
\hline \multicolumn{2}{||c||}{ Parameters of Analysis Without GOOSE } \\
\hline \hline Lifespan & 15 years \\
\hline Investment Value & $\$ 9,200,000,000$ \\
\hline AOM percentage & $2.33 \%$ \\
\hline AOM cost & $\$ 214,360,000$ \\
\hline \hline Annual Savings & $\$ 1,557,793,791$ \\
\hline Minimum expected rate of return & $10.0 \%$ \\
\hline \hline TIR & $11.9 \%$ \\
\hline \hline VPN & $\$ 925,694,754$ \\
\hline \hline Opportunity rate & $1.98 \%$ E.A. \\
\hline \hline
\end{tabular}

TABLE VII. Economic analysis with GOOSE

\begin{tabular}{||l|c||}
\hline \multicolumn{2}{|c|}{ Parameters of Analysis With GOOSE } \\
\hline \hline Lifespan & 15 years \\
\hline Investment Value & $\$ 9,200,000,000$ \\
\hline AOM percentage & $2.33 \%$ \\
\hline AOM cost & $\$ 214,360,000$ \\
\hline \hline Annual Savings & $\$ 1,557,793,791$ \\
\hline \hline Minimum expected rate of return & $10.0 \%$ \\
\hline \hline TIR & $12.3 \%$ \\
\hline \hline VPN & $\$ 1,116,317,712$ \\
\hline \hline Opportunity rate & $1.98 \%$ E.A. \\
\hline \hline
\end{tabular}

\section{Conclusions}

According to the results of the Monte Carlo simulations of Electrical Substation double busbar that are made by Matlab GUI, designed to develop this study, we can observe from the implementation of the IEC61850 standard, that it increases reliability of the system by approximately $15 \%$, that is, the simulated system without the GOOSE implementation has a $72 \%$ reliability and with the implementation of the Standard it has a reliability of approximately $89 \%$.

Based on the reduction of the simulated system failures, the system presents increased reliability, resulting in an increase in yearly economic gains, which is determined by calculating the quality indexes. These indexes show that with the implementation of the standard there is a gain of $\$ 398,085,852.80$ pesos per year, that is, approximately a $15 \%$ gain versus the system without the implementation. 
Taking into account the economic projection, it is said that increasing the IRR, project gains are increased, in this system, not having this standard, the IRR is $11.9 \%$ and with the implementation of the system, a projection of $12.3 \%$ is obtained, so the implementation of the standard is recommended, because it increases the substation economic production annual profit.

\section{REFERENCES}

[1] C. Kriger, S. Behardien, J-C. Retonda, a detailed analysis of the GOOSE message structure in an IEC 61850 standard-based substation automation system, International Journal of Computers Communications and Control, Volume 8, Issue 5, pp.708-721, 2013.

[2] N. Garcia, D. López, J. Herrera, Simulation and analysis of mass interactive flows in a label switching backbone, International Journal of Applied Engineering, Volume 10, Issue 15, pp. 35226-35228, 2015

[3] W. Dickinson, et al, Fundamentals of reliability techniques as applied to industrial power systems, IEEE Conference Technical 71C1816A, pp. 10-31, 1971.

[4] R. Billinton, R. Allan, Reliability evaluation of power systems, Second Edition, Editorial Plenum, New York , 1996.

[5] R. Bono, R. Alexander, A. Dorman, Y-J. Kim, J. Reisdorf, Analyzing reliability - A simple yet rigorous approach, IEEE Transactions on Industry Applications, Volume 40, Issue 4, pp. 950-957, 2004.

[6] Simulación método Monte Carlo, Facultad de Ciencias Exactas, Investigación Operativa I, Universidad del Centro de la Pcia de Buenas Aires, 2005.

[7] J. Burke, Power distribution engineering: fundamentals and applications, First Edition, Copyrighted Material, New York, 1994.

[8] J. Carreño, D. López, O. Salcedo, Criterios y consideraciones metodológicas y tecnológicas a tener en cuenta en el diseño e implementación del protocolo IEC 61850 en la automatización y protección de sistemas de potencia eléctrica, Redes de ingeniería, Volume 3, Issue 1, pp. 23-40, 2012. 